\title{
Environmental and nursing-staff factors contributing to aggressive and violent behaviour of patients in mental health facilities
}

\begin{tabular}{|c|c|}
\hline \multicolumn{2}{|c|}{$\begin{array}{l}\text { Authors: } \\
\text { Evalina van Wijk }{ }^{1} \\
\text { Annalene Traut }{ }^{1} \\
\text { Hester Julie }^{1}\end{array}$} \\
\hline \multicolumn{2}{|c|}{$\begin{array}{l}\text { Affiliations: } \\
{ }^{1} \text { School of Nursing, University } \\
\text { of the Western Cape, } \\
\text { South Africa }\end{array}$} \\
\hline \multicolumn{2}{|c|}{$\begin{array}{l}\text { Correspondence to: } \\
\text { Evalina van Wijk }\end{array}$} \\
\hline \multicolumn{2}{|c|}{$\begin{array}{l}\text { Email: } \\
\text { evalina.vanwijk@ } \\
\text { westerncape.gov.za }\end{array}$} \\
\hline \multicolumn{2}{|c|}{$\begin{array}{l}\text { Postal address: } \\
\text { Western Cape College of } \\
\text { Nursing, Klipfontein Road, } \\
\text { Heideveld 7764, South Africa }\end{array}$} \\
\hline \multicolumn{2}{|c|}{$\begin{array}{l}\text { Dates: } \\
\text { Received: } 13 \text { Oct. } 2012 \\
\text { Accepted: } 25 \text { Mar. } 2014 \\
\text { Published: } 14 \text { Aug. } 2014\end{array}$} \\
\hline \multicolumn{2}{|c|}{$\begin{array}{l}\text { How to cite this article: } \\
\text { Van Wijk, E., Traut, A. \& Julie, } \\
\text { H., 2014, 'Environmental } \\
\text { and nursing-staff factors } \\
\text { contributing to aggressive } \\
\text { and violent behaviour of } \\
\text { patients in mental health } \\
\text { facilities', Curationis 37(1), } \\
\text { Art. \#1122, } 9 \text { pages. http:// } \\
\text { dx.doi.org/10.4102/ } \\
\text { curationis.v37i1.1122 }\end{array}$} \\
\hline \multicolumn{2}{|c|}{$\begin{array}{l}\text { Copyright: } \\
\text { (C) 2014. The Authors. } \\
\text { Licensee: AOSIS } \\
\text { OpenJournals. This work } \\
\text { is licensed under the } \\
\text { Creative Commons } \\
\text { Attribution License. }\end{array}$} \\
\hline \multicolumn{2}{|l|}{ Read online: } \\
\hline 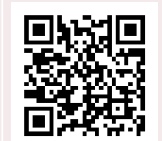 & $\begin{array}{l}\text { Scan this QR } \\
\text { code with your } \\
\text { smart phone or } \\
\text { mobile device } \\
\text { to read online. }\end{array}$ \\
\hline
\end{tabular}

Background: Aggressive and violent behaviour of inpatients in mental health facilities disrupts the therapeutic alliance and hampers treatment.

Objectives: The aim of the study was to describe patients' perceptions of the possible environmental and staff factors that might contribute to their aggressive and violent behaviour after admission to a mental health facility; and to propose strategies to prevent and manage such behaviour.

Research design: A qualitative, phenomenological study was utilised, in which purposefully sampled inpatients were interviewed over a six-month period. Inpatients were invited to participate if they had been admitted for at least seven days and were in touch with reality.

Method: Forty inpatients in two mental health facilities in Cape Town participated in faceto-face, semi-structured interviews over a period of six months. Tesch's descriptive method of open coding formed the framework for the data analysis and presentation of the results. Trustworthiness was ensured in accordance with the principles of credibility, confirmability, transferability and dependability.

Results: Analysis of the data indicates two central categories in the factors contributing to patients' aggressive and violent behaviour, namely, environmental factors and the attitude and behaviour of staff.

Conclusion: From the perspective of the inpatients included in this study, aggressive and violent episodes are common and require intervention. Specific strategies for preventing such behaviour are proposed and it is recommended that these strategies be incorporated into the in-service training programmes of the staff of mental health facilities. These strategies could prevent, or reduce, aggressive and violent behaviour in in-patient facilities.

\section{Introduction}

Aggressive and violent behaviour of inpatients in mental health facilities occurs globally and is a serious and frequent clinical and nursing care problem. A recently-published meta-analysis found 71 reports in the literature dealing with this subject (Papadopoulos et al. 2012). However, at two large state mental health hospitals in Cape Town, it is not clear which factors contribute to such behaviour, as seen from the patients' perspective.

\section{Literature review}

From an international perspective, both qualitative and quantitative studies report that patients' aggressive and violent behaviour is not simply the manifestation of underlying pathology, but can be triggered by factors related to the environment and ward staff (James et al. 1990).

In a study conducted in a closed mental health admission ward in 1997, Nijman et al. (1999) reported one aggressive incident per day. Such incidents are a threat to the safety and wellbeing of patients and nursing staff (Palmstierna \& Wistedt 2000). To minimise the occurrence of aggressive and violent behaviour of patients with mental illness, a therapeutic milieu should be created that promotes their optimal health and well-being in a safe and non-threatening environment (Rawlins, Williams \& Beck 1993).

Conditions in South African mental health facilities, from local to national levels, were placed under the spotlight after 1994, resulting in the promulgation of a new Mental Health Care Act in 2002 (South Africa 2002) (hereafter, 'the Act'). The aim of the Act is to protect patients with mental illness and their belongings; to safeguard the right of every mental healthcare user to respect human dignity and privacy and to prevent any discrimination on the grounds of mental health status (South Africa 2002). 


\section{Problem statement}

As a lecturer responsible for accompanying nursing students, the researcher found that many patients appear to be unhappy with circumstances in their wards, including their interactions with staff. These observations gave rise to the following research question: 'What are the environmental and staff-related factors that could contribute to aggressive and violent behaviour on the part of patients after admission to a mental health facility?'

\section{Aim of the study}

The purpose of the study was to explore and describe patients' perceptions of the possible factors (environmental- and staffrelated) that might contribute to their aggressive and violent behaviour after admission to a mental health facility, as well as to re-contextualise the findings as proposals in order to prevent and manage such behaviour.

\section{Research method and design Design}

In accordance with the aims described above, a qualitative phenomenological study was undertaken. Phenomenology concentrates on the lived experiences of individuals within their life-world, eliciting commonalities and shared meanings (Polit \& Beck 2013).

\section{Materials}

The population for this study was recruited from patients of two pre-discharge wards at each of two state mental health facilities in Cape Town. These pre-discharge wards are 'open', allowing patients unrestricted movement in and out of the ward. The participants reflected the racial groups of the referral areas; thus, they came from the mixed-race, black (predominantly Xhosa) and white communities. At the time of the study, all the patients were receiving medication.

\section{Data collection}

Purposive sampling was used. Participants could be from any cultural background, but had to:

- be 18 years or older

- be admitted for at least seven days

- have expressed an interest in the study topic and shown a willingness to share their views and experiences by means of an audio-taped interview

- be in touch with reality (not psychotic) and understand the purpose of the study

- have signed an informed consent for study participation.

For the selection process, the consulting psychiatrist in charge of a selected ward was requested to identify all the patients who, in his or her opinion, would meet the above participation criteria.

The interview with each participant was guided by a semistructured interview schedule. This schedule comprised a set of open-ended but predetermined questions based on the external and situational models of aggressive and violent behaviour, as described by Duxbury (2002). Field notes were taken to enrich the data collected.

Apart from three interpreter-facilitated interviews conducted in the Xhosa language, all interviews were in English. The interviews took place in an environment free from disturbances and each one lasted for one to two hours. The participants were asked three central questions:

- What do you think are the possible ward factors which may contribute toward patients' aggression and violent behaviour in the ward?

- What do you think are the possible staff factors which may contribute toward such behaviour in the ward?

- What can be done to prevent and manage such behaviour?

At the end of each interview session, the researcher summarised the participant's perceptions and feelings along with the written field notes in order to validate whether the researcher had the same understanding as the participant. Interviews continued until a repetition of themes within the sample demonstrated that data saturation had been reached. On completion, the interviews were transcribed verbatim so as to ensure the accuracy of data collected. The Xhosa interviews were translated and transcribed into English by the interpreter.

Although the intended sample size for this study was initially 25 participants, the researcher went on to interview 40 , comprising 20 patients at each of the study sites, before she was satisfied that data saturation had been reached. This increase was necessary because some patients who had expressed an interest in the study and had given their informed consent, participated poorly during the interview and did not continue beyond about 10 minutes, with the result being that these interviews lacked depth.

\section{Data analysis and interpretation}

Tesch's descriptive method of open coding was used to analyse the interview transcripts and the field notes. The researcher, as well as an external researcher experienced in qualitative data analysis, both coded the same set of data independently, using the protocol described by Tesch (Creswell 2012). The data from patients at both hospitals were coded together and checked by the external coder. The study supervisor checked all the transcripts to ensure that the participants' meanings had not been modified during the analytic process.

Categories, patterns and themes were sorted manually according to the theoretical framework described by Duxbury (2002). Subsequently, an overall thematic description was developed regarding the inpatients' experiences and perceptions of the factors contributing to the behaviour under consideration. Both deductive and inductive reasoning were applied in order to formulate possible strategies for the prevention and management of that behaviour. 


\section{Results}

The ages of the participants ranged from 21 to 55 years. Thirty-two of the participants were unemployed and the highest educational standard was Standard 8 (Grade 10). All 40 participants reported having been involved in, or exposed to, some form of aggression and physical violence in the ward.

Three themes emerged, of which two concerned issues in the ward environment: these two issues were the living conditions and ward atmosphere. The third theme concerned staff interactions (staff andpatient interaction). Some overlap was found amongst the various identified patterns within each theme. The participants' mental illness and the distress caused by being admitted into a mental health facility immediately turned their lives upside down.

\section{Environmental factors \\ Theme 1: Living conditions}

Unhygienic surroundings: After admission to the mental health facility, the participants were propelled immediately into an environment that they never believed could be possible in a hospital. The filthy state of the wards caused much anger and dissatisfaction:

'The place is unhealthy, pathetic ... sometimes the blankets are smelly and torn ... the cockroaches run all over the place.' (P26, Female, 33 years)

Patients perceived that they were being ignored by staff and not listened to, when they complained about the poor and unhygienic state of the environment. This caused frustration amongst participants and escalated in outbursts of anger:

'We reported in a climate meeting that our blankets smell of urine, but the nurses ignored our complaints.' (P1, Male, 41 years)

When asked to suggest strategies to prevent aggressive and violent behaviour, participants said that staff should ensure that all items, including bedding, were clean and tidy; cleaning procedures should be carried out according to the hospital routine and additionally as needed; regular pest control measures should be in place; floors should be washed with water and soap daily; patients should be educated about the prohibitions regarding smoking and eating in the bathrooms; the bathrooms should be provided with rubbish bins; and nursing staff should listen when patients raise their concerns.

The quality and quantity of food: Frustration about the quality and quantity of food caused fear and anxiety, as was described by this participant:

'I am not satisfied with the hospital food; it is often served cold and late ... the quality of the food is poor and it is very little.' (P32, Male, 27 years).

\section{Another participant stated:}

'The kitchen did not make provision for the food preferences of our different cultures and it annoyed me and some of the other patients to eat Western food.' (P2, Male, 49 years)
Unavailability of daily necessities: After being admitted to a mental health facility, participants in the study felt humiliated and were horrified about the unavailability of daily necessities. In addition, they felt that this contributed to the anger and dissatisfaction that they felt during their stay on the ward:

'I was very angry with the nurse because she washed my face with a cloth soiled with BA [faeces].' (P17, Female, 25 years)

Another participant remarked:

'If you are not happy with the way it is here, you must go to a private hospital.' (P12, Female, 54 years)

Participants suggested the following strategies in order to encourage good hygiene habits and reduce patients' frustration levels: staff should ensure that there is an adequate supply of shampoo, facecloths, towels and toilet paper for daily personal care; and they should listen when patients reported these issues.

Lack of privacy: The distress of being admitted into a mental health facility was compounded by the violation of the patients' rights to privacy and the realisation that their personal information was not kept as confidential as they had been told at admission:

'How can I trust the staff with my problems when I heard how they discuss our problems loudly in front of other patients?' (P2, Male, 49 years)

The participants recommended the following strategies in order to avoid negative behaviour: the multidisciplinary team should keep patients' information confidential; patients and nursing staff should be encouraged to respect each other's privacy and private property; and the hospital should provide an area where patients can have some 'alone time' or can have conversations in private with their relatives and friends.

Noise levels: Although the participants realised that they were all in need of treatment, they vacillated between feelings of anger and helplessness about their fellow patients who were noisy and insulted other patients. This resulted in aggressive outbursts and violence, as reported by this participant:

'The constant screaming and fights in the ward make me sicker than when I came here.' (P33, Male, 26 years)

With regard to the noise levels in the ward, the participants suggested that nursing staff should heed patients' complaints about the exceedingly high volume of the TV and music. Furthermore, the patients' cultures and music preferences should be taken into consideration.

Crowding: Participants expressed their dissatisfaction with the circumstances in the ward and described it as a place of locked doors and heavy sealed windows:

'It feels as if I am in a prison ... there is limited space, which I have to share with several other patients.' (P11, Male, 38 years) 
Participants in the study felt overwhelmed, in addition to feeling deprived of their dignity and privacy in the bathrooms:

'In the mornings, we stand like sheep in a row waiting for our turn to have a shower or a bath.' (P28, Male, 51 years)

Negative experiences of seclusion: Of the 40 patients involved in this study, 37 were subjected to seclusion. Seclusion was perceived as being a frightening and overwhelming experience, as was described by this participant:

'It is the most terrible thing when they take off all your clothes and take away your personal belongings.' (P7, Female, 26 years)

Participants expressed their horror about what had happened in the seclusion rooms and expressed a sense of personal vulnerability:

'The nurse smacked me in the face and kicked me in the stomach ... then put me in a seclusion room.' (P18, Female, 35 years)

Participants felt that staff members should demonstrate good interpersonal skills when assisting patients; and that the space allocated for seclusion should be in a safe environment where patients cannot harm themselves.

Unfair limit-setting: Participants vacillated between feelings of satisfaction and intense feelings of unhappiness with some of the ward rules:

'Most of the rules are fair, but we are unhappy with the rules pertaining to the times we have to get up and go to bed.' (P08, Female, 45 years)

Despite the patients' attempts to inform the nursing staff about their unhappiness with the unfair manner in which limits are set, communication between patients and nursing staff evoked unpleasant feelings:

'Keep with the rules or face going back to the locked wards, or get injections.' (P36, Female, 37 years)

The participants proposed that limit-setting be consistent across race groups: whenever possible (in non-secure units), patients should be able to leave the ward; ward rules and their purpose should be explained properly; patients should be involved in the formulation of new ward rules; and unit rules should be more flexible.

Lack of structured activities: The participants expressed concern regarding the lack of structured interaction and socialisation (eg. ward outings, therapeutic groups, interviews), especially over weekends. They felt this aggravated their aggression and violent behaviour:

'Weekends are boring; there are no students or anyone that can talk to us except for the security guard.' (P40, Female, 53 years)

Frustration about the type of activities available was described by this participant:

'I am so fed-up with dominoes, I do not want to see it ever again after I leave this place.' (P16, Male, 29 years)
Unhappiness about the circumstances in the ward after visiting times was described as follows:

'Some patients are likely to become more hostile and aggressive after visiting times because those who did not get any visitors, begged drinks and cigarettes from the patients who did receive visitors and this often caused friction amongst us.' (P18, Female, 35 years)

Circumstances around meal times were described as un pleasant and fearful:

'During the administration of medication in the dining room, certain staff members scream at the patients if they [do] not fetch their medication quickly enough.' (P34, Male, 37 years)

Another remark was:

'Many of patients' aggressive outbursts are provoke[d] during meal times by the presence of other patients with severe sideeffects from the medication.' (P35, Female, 55 years)

Participants suggested that they should have more access to occupational therapy and interesting group activities. In addition, treatment programmes should be made more flexible so as to fit their needs; and staff should be more approachable when patients want to discuss their unhappiness regarding the lack of activities.

\section{Theme 2: Ward atmosphere}

Disrespect for patients' culture, religion and rights: All the study participants volunteered that they had been informed about their rights at the time of admission, including their right to participate in decision making on matters affecting their health. However, this participant reports that the culture, religion and rights of some patients are grossly violated:

'Certain race groups were favoured by staff members of the same race concerning issues of seclusion, after being involved in an aggressive incident and we were very unhappy about this.' (P12, Male, 54 years)

Patients perceived that they were being ignored, not listened to and therefore disrespected; this caused agitation:

'Nursing staff did not always allow us to highlight our problems but told us to hurry up, because they did not have time or were in the mood to listen to our nonsense stories.' (P4, Female, 35 years)

Patients complained about inconsistency of care across wards and different nursing shifts, reporting that some staff did not treat all the patients equally, especially when food and attending activities were concerned. The perceptions of some of the participants imply discrimination on racial grounds:

'If you are black, the coloureds and whites will get their food first; they also get bigger portions than us blacks.' (P45, Female, 24 years)

The study participants suggested that patients be given freedom of speech regarding matters affecting them; they should be accepted unconditionally, irrespective of their behaviour. On admission, patients should be informed of 
their rights. In addition, the integrity of person of all patients must be protected at all times; nursing staff should be made aware of how respect is shown in varying cultures; a patient's cultural background should be considered when their care plan is formulated; and staff should avoid creating situations that humiliate patients, wherein they force them to do anything that goes against their religion (eg. dancing).

Use of medication and injections to keep patients quiet: Regrettably, 30 of the 40 participants perceived sedation as being a mechanism to keep them quiet, rather than to treat them. If they did not behave or act as the nursing staff demanded, they were threatened with seclusion or injections:

'When we are too talkative, or if we ask for medical attention, we are threatened with seclusion or injections.' (P38, Male, 34 years)

The study participants suggested that nursing staff should listen to what patients were saying; and that medication, physical restraint and seclusion should never be used as a threat or punishment.

Smoking habits of patients: Issues regarding smoking were problematic for both smokers and non-smokers. Non-smokers were frustrated because their rights were not protected. For example, they were forced to sit in the same day room as smokers, as was described by this participant:

'There are times when I feel nausea [sic] when I have been sitting next to a person who smokes.' (P33, Male, 26 years)

Furthermore, non-smokers had to share rooms with smoking patients, which often caused anger outbursts:

'Many of the aggressive outbursts in this ward occurred while [the] nursing staff is trying to set limits regarding smoking.' (P15, Female, 38 years)

The participants suggested that a room be provided where smokers could enjoy their cigarettes and that patients' families should be encouraged to provide money or cigarettes for their family members. The smokers felt that nursing staff should protect them from patients who had run out of cigarettes.

Feeling unsafe in the ward: For this participant, the dangerous and unpredictable patients in the ward made him feel unsafe:

'Certain male patients insist on homosexual advances and the night staff was usually not around during these times; and when they were informed about these incidents, they were not interested.' (P22, Male, 27 years)

The participants reported that their fears, along with the feelings of insecurity of the other patients in the ward, led to angry outbursts and disruptive behaviour:

'One doesn't sleep well at night because you never are sure whether your personal belongings will still be on your locker when you get up in the morning. I feel very angry towards the patients in the ward and the nurses who are not respecting our personal belongings, because when I reported that my jacket disappeared from my locker, the nurse was not supportive and said that the dirty linen have already being taken away.' (P33, Male, 26 years)

Participants suggested that staff should be more aware regarding the patients' fear, anxiety, frustration and feelings of intimidation and victimisation by fellow patients and even by certain nursing staff members.

Nursing staff-to-patient ratio: In this study, the nursing staff-patient ratios ranged from 1:8 to 1:9. The participants had mixed views about whether patient behaviour changed according to the number of nursing staff on duty, although there was general agreement that most of the nursing staff were insensitive and not available when they were needed. Incidents were more likely when certain staff members were on duty: those staff had a reputation for provoking anger in patients, no matter how many other staff members were on duty:

'The permanent nursing staff members are rarely visible or available - they tend to sit in the offices chatting on telephones or in the tearoom.' (P2, Male, 49 years)

'Some of the nursing assistants are rude, agitated and intolerant and nursing staff often had no time, or are not in the mood to listen to us; and when we approached these staff, they become very irritated and verbally abusive.' (P24, Female, 36 years)

Participants suggested that nursing staff, whether permanent or temporary, should be more visible; and that patients should not be left alone with inadequately-trained security staff, because they felt that security staff members were not equipped to manage mentally-ill patients.

\section{Nursing-staff factors}

Attitude and behaviour of nursing staff: Participants in the current study mentioned that they were scared by the consistently intolerant, rigid and autocratic attitudes of certain nursing staff members. As a result, they did not feel comfortable discussing their emotions for fear of the reactions of those staff, including threats of seclusion or injections. These fears often elicited anger and assaults:

'When we want to discuss our problems with the nurses, they are always too busy to attend to our problems ... telling us to shut up.' (P25, Female, 36 years)

In this study, participants reported that certain nursing staff members, men as well as women, including agency and permanent staff, were rigid, judgemental and abusive. A participant who was upset about the manner in which her complaint was handled described her experience as follows:

'I voiced my unhappiness and complaints in a letter to the superintendent of the hospital via the nursing staff. After three weeks, I enquired whether the letter was indeed sent to the superintendent. The nurse verbally and physically abused me and sent me back to the closed ward without any explanation. Afterwards I suffered bruising and when I told this nurse that I am aware of my rights, she further abused me.' (P9, Female, 47 years) 
Another participant described the behaviour of these nursing staff members as:

‘ $a$ ] disgrace to the nursing profession ... because they are scolding, shouting and slapping the patients instead of being caring.' (P13, Male, 29 years)

Only a minority of participants perceived the nursing staff as being approachable, pleasant and available to listen to their problems with empathy. One participant highlighted the following interaction that left him feeling cared for:

'What surprised me was that when I was in seclusion, one of the nurses visits [sic] me often to talk to me, which let me not feel so lonely and neglected.' (P14, Male, 27 years)

A general remark was that all nursing staff should communicate with the patients directly and not via security staff. In addition, they should be more available and willing to assist with patients' requests.

\section{Ethical considerations}

Ethics approval was obtained from the Higher Degrees Ethics Committee of the Faculty of Nursing of the University of the Western Cape (Ref. 04/2/21). The managers of both psychiatric hospitals permitted access to the selected wards. Throughout the study, the researcher adhered to the ethical guidelines set out in the Declaration of Helsinki (Reynolds 2000).

All the patients recruited were informed of the purpose of the study. They also gave permission for the use of a tape recorder for data collection during the interviews. Participants were given a guarantee that the taped information would be kept confidential and that the tapes would be destroyed once the interviews had been transcribed. In order to maintain their anonymity, participants were allocated study numbers. Furthermore, they were guaranteed that they were under no obligation to participate and that they had the right to withdraw from the study without being prejudiced in any way (Streubert \& Carpenter 2011).

\section{Trustworthiness}

To ensure trustworthiness of the data, the four principles of credibility, transferability, dependability and confirmability were followed, as outlined by Streubert and Carpenter (2011).

\section{Credibility}

The researcher's interpretation of the data is supported by the participants' own words. The involvement of the study supervisor and an external qualitative researcher further enhanced the validity of the study. Triangulation amongst reliable literature sources was used to re-contextualise the findings for the purpose of formulating preventative management strategies.

\section{Transferability}

Although the study is contextual in nature, the nature of the problem is of concern to all staff rendering patient care in mental health facilities. To guarantee transferability, the inclusion criteria for the study were designed to facilitate recruitment of a typical category of mental health patients whose perceptions could reflect the key issues in the research problem. The study methods are described and references to known literature sources that relate to the identified themes are provided; other researchers are therefore able to transfer or adapt the conclusions of this study to other settings.

\section{Dependability and confirmability}

A clear distinction was maintained between the researcher's personal views and those of the participants, in accordance with the principle of reflexivity. All the participants' verbal and non-verbal cues were recorded by means of a tape recorder and accurate field notes (LeVasseur 2003). The use of an independent external coder, who coded the transcripts independently of the researcher, further increased the dependability and confirmability of the study.

\section{Discussion}

The challenge with regard to understanding, preventing and controlling aggressive and violent behaviour remains a critical issue in in-patient facilities. The current study attempted to identify the environmental and staff factors that contribute to such behaviour, as perceived by a sample of patients at two Cape Town state mental health hospitals. Input from the staff did not form part of the study.

It is clear that the mental illness per se is not the sole cause of the patients' aggressive and violent behaviour. Participants in this study described their experiences in the ward as being 'living torture' and 'depressing'. They believed that their fear, tension, distrust, anger and frustration had a negative impact on their mental health. They were disturbed by the unpleasant atmosphere in the ward and their perceptions revealed that many environmental and situational factors contribute to frustration and a reduced threshold to aggressive outbursts and disruptive, violent behaviour against self, fellow patients and/or staff members.

Most of the participants described their admission to a mental facility as admission to a living hell and viewed the mental facility as being a frightening, negative, punitive, and brutal experience. The study participants shared many concerns which contribute to their aggressive and violent behaviour. These include the unhygienic conditions in the ward; a poor living environment; the tense ward atmosphere; shortages of personal hygiene items such as face cloths, towels, toilet paper, deodorants, razors and toothbrushes; their fellow clients who were demanding, loud and provocative; patients with different diagnoses being accommodated in one ward; and overcrowding and feeling unsafe in the ward. Overcrowding in wards has already been linked with violence, because patients may become over-stimulated as a result of turmoil in the ward (Palmstierna, Huitfeldt \& Wistedt 1991). Other researchers have also recognised various aspects of the ward milieu as being important predictors of treatment 
outcomes and patient satisfaction with service delivery (Middelboe et al. 2001:212).

Violence is less likely to occur in environments where an optimal balance is maintained between density, privacy and control; this can be achieved, for example, by such simple environmental strategies as the allocation of individual rooms and the prevention of crowding (Nijman et al. 1999, 2002). Some of these strategies are also reflected in the suggestions proposed by participants for the prevention of aggressive behaviour as a result of crowding: these include the provision of adequate space in bedrooms, smoking rooms and daily living areas; an increase in the number of nursing staff provided for the supervision of patients; and better control of temperature and ventilation.

It is important to attend to hygiene needs in the therapeutic environment because mentally-ill patients are admitted in varying states of cleanliness, ranging from good to extremely neglected. Many have difficulty with regard to taking responsibility for self-care and need to be supported in their efforts to improve their personal grooming and appearance. Good patient education is therefore a key to good hygiene habits. Morrison Valfre (2001:131) states that a tidy and well-maintained environment sends a message of caring and pride in appearance and suggests that it is the nurses' responsibility to monitor how each of these aspects affects their clients.

Although participants agreed that rules were necessary in order to manage and prevent chaos in the wards, others were extremely dissatisfied about the inconsistent limit-setting styles and perceived these as being both provocative and unacceptable; and these participants mentioned especially a lack of communication about what was expected from them in the ward. This applied particularly when patients had come from other wards that operated according to different rules. Patients who did not attend occupational therapy were frustrated by restrictions on their freedom and said that the ward environment was 'boring, with nothing to do'. Participants perceived an increase in the number of incidents on Monday and Tuesday, with the least on Friday, because some of the patients went on weekend leave.

Issues around food and meals were frequent, with some participants expressing concerns over mealtimes. Although it is not always possible to serve meals as scheduled, the participants suggested that patients be informed by the staff when there would be delays.

Food should be served warm, with preferences and special diets being taken into consideration. Staff members should not be allowed to eat patients' food. It was reported that some of the assistant nurses served up the food and then disappeared. This frustrates some patients and at times they take out their frustrations on the furniture or become violent toward other patients or the staff. The findings of a South African study conducted in a mental health facility suggest that a relaxing atmosphere at mealtimes allows nurses to attend to any problems that might occur, such as patients receiving too little food or cold food. This is because interference with basic human needs, such as food, can lead to fear and anxiety (Lobelo 2004).

This study has highlighted patients' negative experiences of seclusion. The term 'seclusion' refers to the containment of a patient in a room or other enclosed area that does not allow the patient to leave that area. Most of the participants reported they were appalled by the rude way that some nursing staff treated them whilst they were in seclusion. Whilst some of them were managed roughly, others were even denied access to toilet facilities. It is, however, impossible to manage severely emotionally-disturbed patients whose behaviour is aggressive or violent without some form of seclusion or physical or mechanical restraint. These techniques are not only helpful with regard to controlling patients, but are also effective for injury prevention and reduction of agitation (Van der Merwe et al. 2009). Palmstierna and Wistedt (2000) suggested that all nursing staff be trained properly in control and restraint techniques in order to reduce the number of aggression-related incidents. All nursing staff should also be made aware of the legal and ethical issues related to physical management strategies and that they can face charges of misconduct if they neglect patients in their care (South Africa 2002).

Contrary to the the South African Nursing Council Regulations 2598 and 378 as well as the stipulations of the Mental Health Care Act (South Africa 2002; South African Nursing Council 1984), some participants reported that certain nursing staff disrespected their culture and religion. For example, certain racial and religious groups were favoured by staff members belonging to those same groups, which left other patients feeling neglected and frustrated. Another concern reported was that patients who had their own cigarettes were often instructed by nursing staff to share them with others. If they refused, they were threatened with restrictions on smoking. Most participants claimed that it was sometimes better to keep quiet because when they voiced their dissatisfaction about the issues that increase their levels of frustration, certain nursing staff members, men as well as women, including agency and permanent staff, reacted inappropriately. Their reactions included behaviour that could be rigid, provocative, judgemental, abusive, rude or impatient or any combination thereof. It was alleged that staff would be impatient and would victimise, bully and intimidate patients or threaten them with injections, seclusion or a return to the locked wards or delayed discharge. Being ignored and neglected frequently led to conflict and outbursts of anger. The quality of the therapeutic relationship has been recognised as being at the heart of mental health nursing; and the nurse-patient interaction has a significant impact on the patients' wellbeing and the quality and outcome of nursing care (Duxbury \& Whittington 2005).

Davis (1991) has reviewed the issue of patient-staff interaction. When members of staff provoke aggressive behaviour in patients, this may result in negative counter-transference 
reactions amongst staff members, which creates additional problems with regard to therapeutic communication. Davis identified two distinct points of view in this regard: patients generally claimed that their aggressive behaviour was a response to provocation on the part of other patients or staff, whereas the staff typically claimed that there was no reason for the assault. The viewpoint that staff members can provoke aggression may be supported by the observation that staff may be assaulted at a higher rate than patients. Furthermore, certain staff members seem to be attacked repeatedly. Negative interactions between nursing staff and patients may be associated with violence, since patients' levels of anger are higher when associated with non-therapeutic styles of limitsetting (Duxbury \& Whittington 2005). Threats may increase anxiety and could cause regression to maladaptive coping mechanisms (Rawlins et al. 1993).

\section{Limitations of the study}

This study is limited to two mental health facilities in Cape Town; its findings may therefore not be generalisable to other facilities elsewhere.

Although the participants were selected by the psychiatrist in charge of each ward, some potential participants refused to be interviewed because they felt intimidated by the nursing staff, who told them 'not to talk nonsense or say too much' in the interviews. Apart from fear of victimisation, in some cases the language differences and patients' underlying psychopathology might have led to some misunderstanding of the purposes of the study or of what would be expected from the participants. Where this was obvious to the researcher, the next patient on the list was asked to participate. This approach could thus have contributed, theoretically, to a selection bias.

\section{Recommendations}

In view of the far-reaching consequences of patients' aggressive and violent behaviour after admission, the prevention and management of such behaviour should be a priority. Mental health facilities need to ensure that all nursing staff receive appropriate training in this area, accompanied by ongoing in-service training and periodic reassessments of the level of risk for the patient, their fellow patients and nursing staff. These assessments should be based on regular audits of the behaviour problem, as well as a comprehensive review of current hospital policies regarding the management of patients who display aggressive and violent behaviour. A further suggestion is to establish a committee that represents the managers and senior mental health nursing staff of all the psychiatric hospitals in the Western Cape Region, in order to facilitate implementation of the proposed strategies and guidelines.

The South African Nursing Council regulates the nursing profession, exercising control over the education and the scope of practice of registered and auxiliary nurses. All relevant South African training institutions and employers should ensure that the highest standards of practice for psychiatric nurses are maintained. The nurses should take constant care regarding the prevention, recognition and therapeutic management of aggressive and violent behaviour in inpatient facilities.

\section{Conclusion}

Two main categories of issues were identified, after analysing the factors volunteered by study participants as contributing to their aggressive and violent behaviour. The first of these involved environmental factors, such as living conditions and ward atmosphere; the second was the attitude and behaviour of the staff. Aggressive and violent episodes are common and require intervention. Specific strategies for reducing or preventing such behaviour are proposed, with an emphasis on an improvement with regard to patient-staff interaction through in-service training.

\section{Acknowledgements}

This article is based on the first author's mini-thesis at the school of Nursing at the University of the Western Cape: 'An investigation into patients' perceptions of contributing factors toward their aggressive and violent behaviour after admission to a mental health facility', submitted in 2006 in partial fulfilment of the requirements for the degree of Magister Curationis.

\section{Competing interests}

The authors declare that they have no financial or personal relationship(s) which may have inappropriately influenced them in writing this article.

\section{Authors' contributions}

E.v.W. (University of the Western Cape) was the principle investigator and made significant contributions to the research design and literature review, including the collection, analysis and interpretation of the data. In addition, she compiled the article and agreed to be accountable for all revisions. A.T. (University of the Western Cape) checked and edited the manuscript, having been involved with the conceptualisation and the design of the research. H.J. (University of the Western Cape) was involved in critical revision of the manuscript and gave final approval for publication of the manuscript.

\section{References}

Creswell, J.W., 2012, Qualitative inquiry \& research design: Choosing among five approaches, 3rd edn., Sage Publications Inc., Thousand Oaks, CA.

Davis, S., 1991, 'Violence by psychiatric inpatients: A review', Hospital \& Community Psychiatry 42(6), 585-590.

Duxbury, J., 2002, 'An evaluation of staff and patient views of and strategies employed to manage inpatient aggression violence on one mental health unit: A pluralistic design', Journal of Psychiatric and Mental Health Nursing 9(3), 325-337. http:// dx.doi.org/10.1046/j.1365-2850.2002.00497.x

Duxbury, J. \& Whittington, R., 2005, 'Causes and management of patient aggression and violence: Staff and patient perspectives', Journal of Advanced Nursing 50(5), 469-478. http://dx.doi.org/10.1111/j.1365-2648.2005.03426.x

James, D.V., Fineberg, N.A., Shah, A.K. \& Priest, R.G., 1990, 'An increase in violence on an acute psychiatric ward: A study of associated factors', British Journal of Psychiatry 156, 846-852. http://dx.doi.org/10.1192/bjp.156.6.846 
LeVasseur, J.J., 2003, 'The problem of bracketing in phenomenology', Qualitative Health Research 13(3), 408-420. http://dx.doi.org/10.1177/1049732302250337

Lobelo, M.I., 2004, 'Experiences of relapsed psychiatric patients in Mafikeng in the North West Province, MCur thesis, Department of Nursing Science', Johannesburg Rand Afrikaans University, viewed 26 September 2012, from http://hdl.handle. net $/ 10210 / 1720$

Middelboe, T., Schjødt, T., Byrsting, K. \& Gjerris, A., 2001, 'Ward atmosphere in acute psychiatric in-patient care: Patients' perceptions, ideals and satisfaction', Acto Psychiatrica Scandinavica 103(3), 212-219. http://dx.doi.org/10.1034/j.1600 0447.2001.00102.x

Morrison Valfre, M., 2001, Foundations of mental health care, 2nd edn., Mosby, St Louis, MI.

Nijman, H.L.I., á Campo, J.M.L.G., Ravelli, D.P. \& Merckelbach, H.L.G.J., 1999, 'A tentative model of aggression on inpatient psychiatric wards', Psychiatric Services 50(6), 832-834.

Nijman, H.L.I., Merckelbach, H.L.G.J., Evers, C., Palmstierna, T. \& á Campo, J., 2002, 'Prediction of aggression on a locked psychiatric admissions ward', Act Psychiatrica Scandinavica 105(5), 390-395. http://dx.doi.org/10.1034/j.1600 0447.2002.0o426.x

Palmstierna, T., Huitfeldt, B. \& Wistedt, B., 1991, 'The relationship of crowding and aggressive behavior on a psychiatric intensive care unit', Hospital \& Community Psychiatry 42(12), 1237-1240.

Palmstierna, T. \& Wistedt, B., 2000, 'Violence in psychiatry, view-points for standardized research', Acta Psychiatrica Scandinavica 102(2), 79-80. http://dx.doi.org/10.1034/ j.1600-0447.2000.102002079.x
Papadopoulos, C., Ross, J., Stewart, D., Dack, C., James, K. \& Bowers, L., 2012, 'The antecedents of violence and aggression within psychiatric in-patient settings', Acta Psychiatrica Scandiniva 125(6), 425-439. http://dx.doi.org/10.1111/j.1600Acta Psychiatrica Scand
0447.2012.01827.x

Polit, D.F. \& Beck, C.T., 2013, Essentials of nursing research: Appraising evidence for nursing practice, 8th edn., Wolters Kluwer Health/Lippincott Williams and for nursing practice, 8 th
Wilkins, Philadelphia, PA.

Rawlins, R.P., Williams, S.R. \& Beck, C.K., 1993, Mental health-psychiatric nursing: A holistic life-cycle approach, 3rd edn., Mosby, St. Louis, MI.

Reynolds, J., 2000, 'Declaration of Helsinki revised', Journal of the National Cancer Institute 92(22), 1801-1803. http://dx.doi.org/10.1093/jnci/92.22.1801

SA Nursing Council (SANC), 1984, Regulation 2598: Regulations relating to the scope of practice of persons who are registered or enrolled under the Nursing Act, 1978, viewed 26 September 2012, from http://www.sanc.co.za/regulat/Reg-scp.htm

South Africa, Mental Health Care Act 17 of 2002, viewed 25 May 2014, from http:// www.justice.gov.za/legislation/acts/2002-017_mentalhealthcare.pdf

Streubert, H.J. \& Carpenter, D.R., 2011, Qualitative research in nursing: Advancing the humanistic imperative, 5th edn., Wolters Kluwer Health/Lippincott Williams and Wilkins, Philadelphia, PA.

Van der Merwe, M., Bowers, L., Jones, J. \& Muir-Cochrane, E., 2009, 'Seclusion: A literature review' viewed 26 September 2012, from http://www.kcl.ac.uk/iop/ depts/hspr/research/ciemh/.../LitRevSeclusion.pdf 